\title{
Dynamics and Berry phase of two-species Bose-Einstein condensates
}

\author{
Z.-D. Chen, ${ }^{1,2,3}$ J.-Q. Liang, ${ }^{1}$ S.-Q. Shen, ${ }^{4}$ and W.-F. Xie ${ }^{2}$ \\ ${ }^{1}$ Institute of Theoretical Physics and Department of Physics, Shanxi University, Taiyuan, Shanxi 030006, China \\ ${ }^{2}$ Department of Physics, Guangzhou University, Guangzhou, Guangdung 510405, China \\ ${ }^{3}$ Department of Physics, Jinan University, Guangzhou, Guangdung 510632, China \\ ${ }^{4}$ Department of Physics, The University of Hong Kong, Hong Kong, China
}

(Received 27 April 2003; revised manuscript received 10 November 2003; published 18 February 2004)

\begin{abstract}
In terms of exact solutions of the time-dependent Schrödinger equation for an effective giant spin modeled from a coupled two-mode Bose-Einstein condensate (BEC) with adiabatic and cyclic time-varying Raman coupling between two hyperfine states of the BEC, we obtain analytic time-evolution formulas of the population imbalance and relative phase between two components with various initial states, especially the $\mathrm{SU}(2)$ coherent state. We find the Berry phase depending on the number parity of atoms, and particle number dependence of the collapse revival of population-imbalance oscillation. It is shown that self-trapping and phase locking can be achieved from initial $\mathrm{SU}(2)$ coherent states with proper parameters.
\end{abstract}

DOI: 10.1103/PhysRevA.69.023611

PACS number(s): 03.75.Kk, 05.30.Jp, 32.80.Pj, 03.65.Fd

\section{INTRODUCTION}

The experimental discovery of Bose-Einstein condensation in trapped atomic clouds opened up the exploration of quantum mechanics of mesoscopic systems in a qualitatively new regime. The cold gas clouds have many advantages for investigation of quantum phenomena, and hence become a test ground of quantum-mechanical principles as well as the interplay between macroscopic and quantum coherence. The observation of matter-wave interference implies the realization of coherent atomic beams, atomic Josephson effect, and a variety of quantum interference phenomena [1]. In particular, recent experiments on two-component Bose-Einstein condensates (BECs) in ${ }^{87} \mathrm{Rb}$ atoms $[1,2]$ have stimulated considerable interests in the phase dynamics and number fluctuations of the condensates. Aside from its intrinsic appeal, the capability demonstrated by the recent experiments might lead to applications also on quantum computation. Based on the macroscopic wave-function approach it is demonstrated that the Josephson effect exists in a driven twostate single-particle BEC in a single trap. The macroscopic quantum self-trapping, as well as the $\pi$-phase oscillations in which the time-averaged value of the phase difference is equal to $\pi$, has been studied extensively [3-7]. It is also shown that the population oscillation is modulated by the collapses and revivals due to the quantum nature of the system [8-14]. The relative phase of two condensates in different hyperfine atomic states can be measured [2] using Ramsey's method of separated oscillating fields [15] and it is evident that the phase locking indeed occurs for small separation between condensates [1], implying the broken gauge symmetry. Most theoretical studies are focused on semiclassical analysis and a full quantum-mechanical formulation of the dynamics of the two-component BECs coupled by timedependent driving is certainly of interest and importance. It is well known that the system of two-component BECs can be described by a giant or mesoscopic pseudospin [16] using Schwinger realization of angular momentum operators in terms of two-mode bosons. Berry phase [17] emerges naturally in the mesoscopic pseudospin model [16] if the cou- pling between two components varies with time cyclically and adiabatically. The Berry phase in the mesoscopic spin model for the coupled two-component BECs has been explored recently in an elegant way by means of geometric evolutions [16]. Trapped atomic BECs make it possible to create mesoscopic quantum objects containing of the order $10^{6}$ atoms in the same quantum state with a longer lifetime, allowing the implementation of adiabatic evolution which is required for the Berry Phase. However, the dynamics of the mesoscopic spin modeled from the two-mode BEC has not yet been studied in the quantum-mechanical formalism. We in the present paper use the exact solution of the timedependent Schrödinger equation for the mesoscopic spin to provide a quantum-mechanical evaluation of the phase dynamics and the number fluctuation. With the time-evolution operator obtained by means of the generator of timedependent SU(2) coherent states [18] we are able to derive analytic time-evolution formulas of both population imbalance and relative phase between the two-component BECs for various initial states, in particular, the $\mathrm{SU}(2)$ coherent state with which the new effect of particle number dependence is discovered. Moreover, our approach has the advantage to obtain the phase dynamics and number fluctuation for both cases with and without the nonlinear interatomic collisions in the same framework so that the effects of interatomic collision can be recognized explicitly by comparing the results between two cases. We show that interatomic collisions do not affect the Berry phase but lead to the damping and collapse revival of the population-imbalance oscillation depending explicitly on the coupling strength and the total number of atoms as well. The SU(2) coherent states are the most realistic initial states for the two-species BEC created by coupling two hyperfine states of atoms with radiation field [12]. To our knowledge, we in this paper report for the first time a full quantum-mechanical evaluation of the dynamics of the two-species BEC described by an explicitly timedependent Hamiltonian with the initial SU(2) coherent states and the phenomena such as self-trapping, phase locking, and collapse-revival are recovered theoretically in the same formalism. 
The plan of the paper is as follows. In Sec. II we derive the mesoscopic spin model from the two-component BECs. The SU(2) coherent states, which are considered as most practical initial states for the two-species BEC, are briefly introduced. The dynamics and Berry phase of the pseudospin are investigated in terms of the SU(2) coherent-state technique for both cases with and without the interatomic collisions in Sec. III.

\section{MODEL AND INITIAL STATE}

We consider the Bose-Einstein condensate of trapped atomic gas in a single trap consisting of two internal states which are coupled by a spatially uniform radiation field with a Rabi frequency $\Lambda$. Atoms are subjected to trapping potential $V_{l}(l=a, b)$. The atoms interact via elastic two-body collisions with the interaction potential of $\delta$-function-type. In the formalism of second quantization, the system is described by the Hamilton operator

$$
\begin{gathered}
\hat{H}=\sum_{l=a, b} \hat{H}_{l}+\hat{H}_{i n t}+\hat{H}_{f} \\
\hat{H}_{l}=\int d^{3} \mathbf{r}\left\{\hat{\Psi}_{l}^{\dagger}(\mathbf{r})\left[-\frac{\hbar^{2}}{2 m} \Delta^{2}+V_{l}(\mathbf{r})\right] \hat{\Psi}_{l}(\mathbf{r})\right. \\
\left.+\frac{q_{l}}{2} \hat{\Psi}_{l}^{\dagger}(\mathbf{r}) \hat{\Psi}_{l}^{\dagger}(\mathbf{r}) \hat{\Psi}_{l}(\mathbf{r}) \hat{\Psi}_{l}(\mathbf{r})\right\} \\
\hat{H}_{i n t}=q_{a, b} \int d^{3} \mathbf{r} \hat{\Psi}_{a}^{\dagger}(\mathbf{r}) \hat{\Psi}_{b}^{\dagger}(\mathbf{r}) \hat{\Psi}_{a}(\mathbf{r}) \hat{\Psi}_{b}(\mathbf{r}) \\
\hat{H}_{f}=\Lambda(t) \int d^{3} \mathbf{r}\left[\hat{\Psi}_{a}^{\dagger}(\mathbf{r}) \hat{\Psi}_{b}(\mathbf{r}) e^{i \varphi(t)}+\hat{\Psi}_{b}^{\dagger}(\mathbf{r}) \hat{\Psi}_{a}(\mathbf{r}) e^{-i \varphi(t)}\right] .
\end{gathered}
$$

Here we have used the field interaction representation in rotating frame. The Rabi frequency $\Lambda(t)$ is time dependent in the sense that it can be turned on and off adiabatically [3]. The phase $\varphi(t)$ due to the small detuning of external field from resonance excitation varies with time slowly and therefore we work on the adiabatically time-varying Hamiltonian. The phase $\varphi(t)$ we see plays a central role in generating of the Berry phase.

In the two-mode approximation of condensation such that $\hat{\Psi}_{a}(\mathbf{r}) \approx \hat{a} \phi_{a}(\mathbf{r}), \hat{\Psi}_{b}(\mathbf{r}) \approx \hat{b} \phi_{b}(\mathbf{r})$, where $\hat{a}, \hat{b}$ are the annihilation operators obeying the usual boson commutation relations, we have

$$
\begin{aligned}
& \hat{H}_{a}=\omega_{a} \hat{a}^{\dagger} \hat{a}+\frac{\eta_{a}}{2} \hat{a}^{\dagger} \hat{a}^{\dagger} \hat{a} \hat{a}, \\
& \hat{H}_{b}=\omega_{b} \hat{b}^{\dagger} \hat{b}+\frac{\eta_{b}}{2} \hat{b}^{\dagger} \hat{b}^{\dagger} \hat{b} \hat{b}
\end{aligned}
$$

$$
\omega_{l}=\int d^{3} \mathbf{r} \phi_{l}^{*}(\mathbf{r})\left[-\frac{\hbar^{2}}{2 m} \Delta^{2}+V_{l}(\mathbf{r})\right] \phi_{l}(\mathbf{r}), \quad l=a, b,
$$

and

$$
\eta_{l}=q_{l} \int d^{3} \mathbf{r}\left|\phi_{l}(\mathbf{r})\right|^{4}, \quad l=a, b .
$$

The interaction operator between two species of atoms is

$$
\hat{H}_{i n t}=\chi \hat{a}^{\dagger} \hat{a} \hat{b}^{\dagger} \hat{b}
$$

where

$$
\chi=q_{a, b} \int d^{3} \mathbf{r}\left|\phi_{a}(\mathbf{r})\right|^{2}\left|\phi_{b}(\mathbf{r})\right|^{2} .
$$

The transition operator induced by the external field is

$$
\hat{H}_{f}=G(t)\left(\hat{a}^{\dagger} \hat{b} e^{i \varphi(t)}+\hat{b}^{\dagger} \hat{a} e^{-i \varphi(t)}\right)
$$

with

$$
G(t)=\Lambda(t) \int d^{3} \mathbf{r} \phi_{a}^{*}(\mathbf{r}) \phi_{b}(\mathbf{r}) .
$$

The time-dependent coupling drive is characterized by its Rabi frequency $\Lambda(t)$ and the phase $\varphi(t)$. Here we consider the adiabatically varying phase $\varphi(t)$ such that its time derivative is negligibly small. The study of the dynamics of the system would be greatly simplified by introducing of the pseudoangular-momentum operators in terms of Schwinger relation,

$$
\begin{aligned}
& \hat{J}_{x}=\frac{1}{2}\left(\hat{a}^{\dagger} \hat{b}+\hat{b}^{\dagger} \hat{a}\right), \\
& \hat{J}_{y}=\frac{1}{2 i}\left(\hat{a}^{\dagger} \hat{b}-\hat{b}^{\dagger} \hat{a}\right), \\
& \hat{J}_{z}=\frac{1}{2}\left(\hat{a}^{\dagger} \hat{a}-\hat{b}^{\dagger} \hat{b}\right) .
\end{aligned}
$$

The Casimir invariant is

$$
\widehat{J^{2}}=\frac{\hat{N}}{2}\left(\frac{\hat{N}}{2}+1\right)
$$

where $\hat{N}=\hat{a}^{\dagger} \hat{a}+\hat{b}^{\dagger} \hat{b}$ is the total number operator, which is a conserved quantity and thus is set equal to the total number of atoms $N=2 j$ with $j$ being the quantum number of angular momentum. The Hamilton operator apart from a trivial constant reads

$$
\hat{H}=\omega_{0} \hat{J}_{z}+q \hat{J}_{z}^{2}+G(t)\left(\hat{J}_{+} e^{i \varphi(t)}+\hat{J}_{-} e^{-i \varphi(t)}\right),
$$

where $\omega_{0}=\omega_{a}-\omega_{b}+(N-1)\left(\eta_{a}-\eta_{b}\right) / 2, \quad q=\left(\eta_{a}+\eta_{b}\right) / 2$ $-\chi$, and $\hat{J}_{ \pm}=\hat{J}_{x} \pm i \hat{J}_{y}$.

The relative phase of the two-mode BEC surely can be abstracted from the expectation value of the angular momen-

with 
tum operators $\hat{J}_{ \pm}$, which along with the expectation value of $\hat{J}_{z}$ (giving rise to the population imbalance between two components of BECs) is a measurable quantity in experiment.

Since the two-component BECs are experimentally created by coupling two hyperfine states with radiation field, it has been shown that the prepared initial state can be a particular case of the SU(2) coherent state [12] (or known as atomic coherent state in quantum optics) which describes a state with a well-defined relative phase between the two species. However, a full quantum evaluation of the dynamics for the two-species BECs coupled by time-dependent driving with the initial spin coherent states has not yet been given. The $\mathrm{SU}(2)$ coherent state is defined as

$$
\hat{J} \cdot \mathbf{n}|\mathbf{n}\rangle=j|\mathbf{n}\rangle,
$$

where $\mathbf{n}=(\sin \theta \cos \phi, \sin \theta \sin \phi, \cos \theta)$ is a unit vector. The $\mathrm{SU}(2)$ coherent state can be generated from an extreme Dicke state such that

$$
\hat{\Omega}(\theta, \phi)|j, j\rangle=|\mathbf{n}\rangle,
$$

where

$$
\hat{\Omega}(\theta, \phi)=e^{\theta / 2\left(\hat{J}_{-} e^{i \phi_{-}} \hat{J}_{+} e^{-i \phi}\right)} .
$$

This is called the coherent state in the north pole gauge, as compared to the generation of the coherent state from the extreme state $|j,-j\rangle$ where it is called the state in the south pole gauge. The Dicke states are defined as usual $\hat{J}_{z}|j, m\rangle$ $=m|j, m\rangle$ and can be generated from the vacuum by boson creation operators, i.e.,

$$
|j, m\rangle=\frac{1}{\sqrt{(j+m) !(j-m) !}}\left(\hat{a}^{\dagger}\right)^{j+m}\left(\hat{b}^{\dagger}\right)^{j-m}|0\rangle .
$$

The coherent state of Eq. (16) can be expanded in terms of the Dicke states,

$$
|\mathbf{n}\rangle=\sum_{m=-j}^{j}\left(\begin{array}{c}
2 j \\
j+m
\end{array}\right)^{1 / 2}\left(\begin{array}{c}
\cos \frac{\theta}{2}
\end{array}\right)^{j+m}\left(\sin \frac{\theta}{2}\right)^{j-m} e^{i(j-m) \phi}|j, m\rangle .
$$

It is easy to verify that

$$
\begin{gathered}
\left\langle\hat{J}_{z}\right\rangle=\left\langle\mathbf{n}\left|\hat{J}_{z}\right| \mathbf{n}\right\rangle=\frac{N}{2} \cos \theta, \quad\left\langle\hat{J}_{+}\right\rangle=\frac{N}{2} \sin \theta e^{i \phi}, \\
\left\langle\hat{J}_{-}\right\rangle=\frac{N}{2} \sin \theta e^{-i \phi}
\end{gathered}
$$

and the phase $e^{i \phi}$ is seen to be the relative phase of the two species prepared in the initial SU(2) coherent state. In this paper the generator of $\mathrm{SU}(2)$ coherent states Eq. (17) is used as a unitary transformation to formulate the dynamics of the mesoscopic spin system modeled from the two-component BECs.

\section{DYNAMICS AND BERRY PHASE}

\section{A. The case of $q=0$}

We first of all consider the case of $q=0$ achieved by the condition $\left(\eta_{a}+\eta_{b}\right) / 2=\chi$, which, although a special case, is practical in the range of BEC parameters. The equality $q_{a}$ $\approx q_{b} \approx q_{a b}$ can be fulfilled practically for the two-component BECs consisting of ${ }^{87} \mathrm{Rb}$ atoms with different internal states since the scattering lengths of atoms with the two internal states are known at the $1 \%$ level to be [2] in proportion $a_{a}: a_{a b}: a_{b}=1.03: 1: 0.97$, where $a_{a}$ and $a_{b}$ are the samespecies scattering lengths and $a_{a b}$ is the scattering length for interspecies collisions. The ground-state wave function is same, $\phi_{a}(\mathbf{r})=\phi_{b}(\mathbf{r})$, and therefore the condition $\eta_{a} \approx \eta_{b}$ $\approx \chi$ can be satisfied. The model in the case of $q=0$ is exactly solvable. We start with a generalized gauge transformation [19] in terms of the time-dependent unitary transformation [20] defined by

$$
\hat{R}(t)=e^{\lambda / 2\left(\hat{J}_{-} e^{-i \varphi(t)}-\hat{J}_{+} e^{i \varphi(t)}\right)},
$$

which has the same form as the generator Eq. (17) of SU(2) coherent states and is the key point of the present formulation. The time-dependent Schrödinger equation is covariant under the gauge transformation [19] such that

$$
i \frac{d}{d t}\left|\psi^{\prime}(t)\right\rangle=\hat{H}^{\prime}\left|\psi^{\prime}(t)\right\rangle
$$

where

$$
\hat{H}^{\prime}=\hat{R} \hat{H} \hat{R}^{\dagger}-i \hat{R} \frac{\partial}{\partial t} \hat{R}^{\dagger}, \quad\left|\psi^{\prime}(t)\right\rangle=\hat{R}|\psi(t)\rangle
$$

and the state $|\psi(t)\rangle$ is assumed to be the solution of original Schrödinger equation

$$
i \frac{d}{d t}|\psi(t)\rangle=\hat{H}|\psi(t)\rangle
$$

The auxiliary parameter $\lambda$, which is time dependent in general, is to be determined by requiring that the Hamilton operator $\hat{H}^{\prime}$ is diagonal in the $\hat{J}_{z}$ representation. Using the relations given in the Appendix [Eqs. (A1)-(A4)] [20], and noticing the adiabatic condition that $d \varphi / d t \simeq 0$ and $d \lambda / d t$ $\simeq 0$, we obtain the Hamilton operator

$$
\hat{H}^{\prime}=\alpha(t) \hat{J}_{z}, \quad \alpha(t)=\sqrt{\omega_{0}^{2}+4 G^{2}(t)}
$$

with auxiliary parameter $\lambda$ chosen as

$$
\sin \lambda=-\frac{2 G(t)}{\omega_{0}} \cos \lambda
$$

and

$$
\cos \lambda=\frac{\omega_{0}}{\alpha(t)}
$$


It is easy to obtain the exact general solution of the original Schrödinger equation

$$
|\psi(t)\rangle=\sum_{m=-j}^{j} c_{m} e^{-i \alpha_{m}(t)}|j, m(t)\rangle, \quad|j, m(t)\rangle=\hat{R}^{\dagger}(t)|j, m\rangle
$$

with $|j, m\rangle$ being the usual eigenstate of angular momentum $\hat{J}_{z}$ so that $\hat{J}_{z}|j, m\rangle=m|j, m\rangle$. The total phase is seen to be

$$
\alpha_{m}(t)=\varepsilon_{m}(t)+\gamma_{m}(t)=m \int_{0}^{t} \alpha\left(t^{\prime}\right) d t^{\prime},
$$

which consists of the dynamical part given by

$$
\varepsilon_{m}(t)=\int_{0}^{t}\left\langle j, m\left(t^{\prime}\right)|\hat{H}| j, m\left(t^{\prime}\right)\right\rangle d t^{\prime}
$$

and the geometric part, i.e., the Berry phase

$$
\gamma_{m}(t)=-i \int_{0}^{t}\left\langle j, m\left(t^{\prime}\right)\left|\frac{\partial}{\partial t^{\prime}}\right| j, m\left(t^{\prime}\right)\right\rangle d t^{\prime},
$$

which is defined in the usual way. In the following we only consider a time-independent Rabi frequency $G$ for the sake of simplicity. For a variation of one period $T$, i.e., $\varphi(T)$ $-\varphi(0)=2 \pi$, the Berry phase is found as

$$
\gamma_{m}(T)=-m \oint(1-\cos \lambda) d \varphi=m \frac{\omega_{0}-\alpha}{\alpha} 2 \pi,
$$

which has an obvious, geometric meaning from the viewpoint of differential geometry that the one form $d \varphi$ is exact but not closed, where $\alpha=\sqrt{\omega_{0}^{2}+4 G^{2}}$, which is a timeindependent parameter. The Berry phase does not depend on an explicit form of the function $\varphi(t)$ and is simply $m$ times of a solid angle with the polar angle $\lambda$, in agreement with the recent result reported in Ref. [16] in which the Berry phase is evaluated in terms of geometric evolutions for the coupled two-component BECs. We, however, following the original procedure of Berry [17], obtain the Berry phase and the exact wave function as well by solving the time-dependent Schrödinger equation. In our approach the time evolution of both population imbalance and relative phase between two components of BECs can be investigated analytically. The explicit dependence of the Berry phase on the parameters of two-species BECs is also given with our procedure and the properties of the Berry phase can be explored. The geometric phase is actually the same as obtained in the context of SU(2) coherent-state path integrals [21]. To study the dynamics of the mesoscopic spin it is useful to derive the explicit time-evolution operator such that

$$
|\psi(t)\rangle=\hat{U}(t)|\psi(0)\rangle,
$$

where the time-evolution operator is found from the exact general solution of the time-dependent Schrödinger equation $|\psi(t)\rangle$ in Eq. (28) as [20]

$$
\hat{U}(t)=\hat{R}^{\dagger}(t) e^{-i \alpha t \hat{J}_{z}} \hat{R}(0) .
$$

For a given initial state $|\psi(0)\rangle$ of the system the population imbalance between two components can be evaluated by

$$
\Delta N(t)=N_{a}(t)-N_{b}(t)=2\left\langle\psi(0)\left|\hat{J}_{z}(t)\right| \psi(0)\right\rangle,
$$

where the time-dependent angular momentum operator $\hat{J}_{z}(t)$ in the Heisenberg picture is given in the Appendix [Eq. (A5)] with the help of Eqs. (A1)-(A4). For the sake of simplicity, we have set the initial phase to zero, i.e., $\varphi(0)=0$. To have the phase dynamics we need also the time-dependent angular momentum $\hat{J}_{+}(t)$ or $\hat{J}_{-}(t)$ in the Heisenberg picture and the explicit formula of $\hat{J}_{+}(t)$ is shown in Eq. (A6). The time evolution of population imbalance and the expectation value of $\hat{J}_{+}(t)$ given by

$$
\left\langle\hat{J}_{+}\right\rangle=\left\langle\psi(0)\left|\hat{J}_{+}(t)\right| \psi(0)\right\rangle
$$

are evaluated for various initial states as follows.

(1) We consider, first of all, the initial state $|\psi(0)\rangle_{1}$ $=|j, m\rangle$ and obtain in terms of Eqs. (A5) and (A6)

$$
\Delta N_{1}(t)=\frac{2 m}{\alpha^{2}}\left[\omega_{0}^{2}+4 G^{2} \cos \{\alpha t+\varphi(t)\}\right]
$$

and

$$
\begin{aligned}
\left\langle\hat{J}_{+}\right\rangle_{1}= & m \sin \lambda\left[-\cos \lambda e^{-i \varphi(t)}+\cos ^{2} \frac{\lambda}{2} e^{i \alpha t}\right. \\
& \left.-\sin ^{2} \frac{\lambda}{2} e^{-i[2 \varphi(t)+\alpha t]}\right] .
\end{aligned}
$$

The population imbalance exhibits a simple oscillation. It is interesting to see a fact that the state of vanishing imbalance can be achieved for the case of even number of particles (i.e., $j=N / 2$ is integer) with the initial state of $m=0$, but not for the case of odd number of particles where $j$ is half-integer and the state of $m=0$ does not exist.

(2) For a general SU(2) coherent state $|\psi(0)\rangle_{2}=|\mathbf{n}\rangle$, the population imbalance is found as

$$
\begin{aligned}
\Delta N_{2}(t)= & \frac{N}{2}\left(\left\{\cos ^{2} \lambda+\sin ^{2} \lambda \cos [\alpha t+\varphi(t)]\right\} \cos \theta\right. \\
& -\sin \lambda \cos \lambda \sin \theta \cos \phi+\left[\cos ^{2} \frac{\lambda}{2} \cos [\alpha t+\varphi(t)\right. \\
& \left.\left.+\phi]-\sin ^{2} \frac{\lambda}{2} \cos [\alpha t+\varphi(t)-\phi]\right] \sin \lambda \sin \theta\right) .
\end{aligned}
$$

The self-trapping with nonvanishing population imbalance takes place for the initial state with $\phi=0$ and $\theta=-\lambda$. The population imbalance thus reduces to 


$$
\Delta N_{2}(t ; \phi=0, \theta=-\lambda)=\frac{N}{2} \cos \lambda=\frac{N}{2} \frac{\omega_{0}}{\alpha} .
$$

The asymmetric trap potential, i.e., nonvanishing $\omega_{0}$ is the necessary condition to achieve the self-trapping. The expectation value of angular momentum operator $\hat{J}_{+}(t)$ for the initial $\mathrm{SU}(2)$ coherent state is seen to be

$$
\begin{aligned}
\left\langle\hat{J}_{+}\right\rangle_{2}= & \frac{N}{2}\left\{\operatorname { s i n } \lambda \left[\cos ^{2} \frac{\lambda}{2} e^{i \alpha t}-\sin ^{2} \frac{\lambda}{2} e^{-i[\alpha t+2 \varphi(t)]}\right.\right. \\
& \left.-\cos \lambda e^{-i \varphi(t)}\right] \cos \theta+\left[\cos ^{4} \frac{\lambda}{2} e^{i \alpha t}\right. \\
& \left.+\sin ^{4} \frac{\lambda}{2} e^{-i[\alpha t+2 \varphi(t)]}+\frac{1}{2} \sin ^{2} \lambda e^{-i \varphi(t)}\right] e^{i \phi} \sin \theta \\
& +\left[-\cos ^{2} \frac{\lambda}{2} \sin ^{2} \frac{\lambda}{2}\left(e^{i \alpha t}+e^{-i[\alpha t+2 \varphi(t)]}\right)\right. \\
& \left.\left.+\frac{1}{2} \sin ^{2} \lambda e^{-i \varphi(t)}\right] e^{-i \phi} \sin \theta\right\}
\end{aligned}
$$

and reduces to

$$
\left\langle\hat{J}_{+}\right\rangle_{2}(\phi=0, \theta=-\lambda)=N \frac{G}{\alpha} e^{-i \varphi(t)}
$$

for the initial state with $\phi=0$ and $\theta=-\lambda$, indicating obviously the phase locking. We see that self-trapping of both population imbalance and relative phase of the twocomponent BECs can be obtained simultaneously from the $\mathrm{SU}(2)$ coherent state. For the particular case of symmetric trap potential $\omega_{a}=\omega_{b}\left(\omega_{0}=0\right)$ we have $\lambda=-\pi / 2$ seen from Eq. (27), and the Berry phase then reduces to

$$
\gamma_{m}\left(T, \omega_{0}=0\right)=m \oint d \varphi=m 2 \pi .
$$

The population imbalance is

$$
\Delta N_{1}\left(t, \omega_{0}=0\right)=m \cos [2 G t+\varphi(t)]
$$

and

$$
\left\langle\hat{J}_{+}\right\rangle_{1}\left(\omega_{0}=0\right)=m\left[\frac{1}{2} e^{i 2 G t}-\frac{1}{2} e^{-i[2 \varphi(t)+2 G t]}\right] .
$$

The population imbalance for the initial SU(2) coherent state vanishes as seen obviously from Eq. (40) in the case of $\omega_{0}$ $=0$, while

$$
\left\langle\hat{J}_{+}\right\rangle_{2}\left(\phi=0, \theta=-\lambda, \omega_{0}=0\right)=\frac{N}{2} e^{-i \varphi(t)} .
$$

The relative phase of two components is locked exactly to the phase of external field. The phase locking, remains in the case with interatom collisions, i.e., the nonvanishing $q$. It may be worthwhile to emphasize that the Berry phase of Eq. (43) is trivial in the case of an even number of particles ( $m$ is integer) while it would lead to an antiperiodic wave function under $2 \pi$ evolution of the phase angle $\varphi$ for the odd number of particles ( $m$ is half-integer in this case) similar to the spin parity effect in the macroscopic quantum coherence in spin systems [22-24].

\section{B. With nonlinear interactions}

We now consider the general case with nonvanishing but small $q$ for the practical model at hand, i.e., the two-species BEC created by coupling two hyperfine states of ${ }^{87} \mathrm{Rb}$ atoms. For a single trap a reasonable condition is $\omega_{0}=0(\lambda$ $=-\pi / 2$ ). With the help of time-dependent unitary transformation, Eq. (21), and the relation given by

$$
\begin{aligned}
\hat{R} \hat{J}_{z}^{2} \hat{R}^{\dagger}= & \hat{J}_{z}^{2} \cos ^{2} \lambda+\frac{1}{2}\left[\left(\hat{J}_{z} \hat{J}_{+}+\hat{J}_{+} \hat{J}_{z}\right) e^{i \varphi(t)}\right. \\
& \left.+\left(\hat{J}_{z} \hat{J}_{-}+\hat{J}_{-} \hat{J}_{z}\right) e^{-i \varphi(t)}\right] \cos \lambda \sin \lambda+\frac{1}{4}\left(\hat{J}_{+}^{2} e^{i 2 \varphi(t)}\right. \\
& \left.+\hat{J}_{+} \hat{J}_{-}+\hat{J}_{-} \hat{J}_{+}+\hat{J}_{-}^{2} e^{-i 2 \varphi(t)}\right) \sin ^{2} \lambda
\end{aligned}
$$

we obtain, apart from a trivial constant,

$$
\hat{H}^{\prime}=-G \hat{J}_{z}-\frac{q}{2} \hat{J}_{z}^{2},
$$

where the two-photon transition terms (proportional to $\hat{J}_{+}^{2} e^{i 2 \varphi(t)}$ and $\left.\hat{J}_{-}^{2} e^{-i 2 \varphi(t)}\right)$ have been neglected as a reasonable approximation $[13,14]$ which is good enough for the small $q$ in comparing with the transition coupling between two components, namely, $q \ll G$. The Berry phase in this case is the same as Eq. (43) due to $\omega_{0}=0$. The interatom collisions do not affect the Berry phase. The time-evolution operator is

$$
\hat{U}_{q}\left(-\frac{\pi}{2}, t\right)=\hat{R}^{\dagger}\left(-\frac{\pi}{2}, t\right) \exp \left[i t\left(G \hat{J}_{z}+\frac{q}{2} \hat{J}_{z}^{2}\right)\right] \hat{R}\left(-\frac{\pi}{2}, 0\right) .
$$

The population imbalance for the initial state $\left|\psi_{1}(0)\right\rangle$ $=|j, j\rangle$ is possible to be evaluated with the help of Eq. (16) and the Dicke-state representation of the $\mathrm{SU}(2)$ coherent state Eq. (19) as

$$
\begin{aligned}
\Delta N_{1}(q, t)= & \left(\frac{1}{2}\right)^{N} \sum_{m=-N / 2}^{N / 2-1}(-1)^{N-2 m}\left(\begin{array}{c}
N \\
\frac{N}{2}+m+1
\end{array}\right) \\
& \times\left(\frac{N}{2}+m+1\right)^{1 / 2} \\
& \times \cos \left\{\left[G+q\left(m+\frac{1}{2}\right)\right] t-\varphi(t)\right\} .
\end{aligned}
$$

To compare the time evolution of population imbalance obtained here with that in the case of $q=0$, i.e., Eq. (44) (for $m=j=N / 2$ ) where the time variation of imbalance is a simple oscillation, the time evolution of Eq. (50) is shown in Fig. 1 with various values of the ratio $q / G$ and the number of particles $N$. It is seen that the damping goes faster when the 
number of particles, $N$, and the ratio $q / G$ increase [Figs. 1(a,b)]. The $N$ dependence of the oscillation of population imbalance for fixed ratio $q / G$ is shown in Fig. 1(a) for $N$ $=10^{2}$ (dashed line), $N=10^{3}$ (dotted line), and $N=10^{4}$ (solid line), respectively. The nonlinear interaction dependence for a fixed number of particles, $N=10^{3}$, is shown in Fig. 1(b) for $q / G=0.01 \sim 0.1$. With the similar method the time-evolution of population imbalance from the initial spin coherent state Eq. (16), $\left|\psi_{2}(0)\right\rangle=|\mathbf{n}\rangle$, with nonvanishing $q$ is obtained as

$$
\begin{aligned}
\Delta N_{2}(q, t)= & -\sum_{m=-N / 2}^{N / 2-1}\left(\begin{array}{c}
N \\
\frac{N}{2}+m+1
\end{array}\right)\left(\frac{N}{2}+m+1\right)^{1 / 2} \\
& \times \cos ^{N+2 m+1}\left(\frac{-\pi / 2+\theta}{2}\right) \\
& \times \sin ^{N-2 m-1}\left(\frac{-\frac{\pi}{2}+\theta}{2}\right) \\
& \times \cos \left\{\left[G+q\left(m+\frac{1}{2}\right)\right] t-\phi-\varphi(t)\right\},
\end{aligned}
$$

where we again use the Dicke-state representation of SU(2) coherent state Eq. (19). The oscillation of population imbalance is shown in Fig. 2 with various values of the coupling strength $q / G$ and angle $\theta$ [for the sake of simplicity we set $\phi=\varphi(t)=0]$. Besides the damping, the most important effect of the nonlinear interaction with initial spin coherent state is the collapse revival of the population-imbalance oscillation. The particle number dependence of collapse revival is shown in Fig. 2(a) for fixed $q / G$ and the parameter $\theta$. We observe an interesting phenomenon that the frequency of the collapse revival depends on both the number of particles and the coupling strength $q / G$. In Figs. 2(b,c) we show the frequency behavior of the collapse revival varying with the product of the particle number and the coupling strength, $N q / G$. The frequency is almost the same for the same value of the product $N q / G$ while different individual values of $N$ and $q / G$. Moreover $N q / G$ dependence of collapse-revival frequency is not monotonic. A critical value $N q / G=65$ is found at which the frequency of collapse revival approaches a minimum. To see the effect of nonlinear interatom collisions closely we look at the population imbalance Eq. (39) with $\phi=\varphi(t)=0$ for the case of $q=0$ as a comparison. In that case the population imbalance of Eq. (39) reduces to a simple oscillation such that

$$
\Delta N_{2}\left(t, \omega_{0}=0\right)=\frac{N}{2} \cos \theta \cos (2 G t)
$$

for $\omega_{0}=0$. It is obvious that the nonlinear interaction results in both damping and collapse revival of populationimbalance oscillation. The simple oscillation of Eq. (52) with Rabi frequency is in agreement with the experimental observation [2].

Particularly, the initial state can be prepared such that $\theta$ $=\pi / 2$, the population imbalance vanishes for both cases (a)

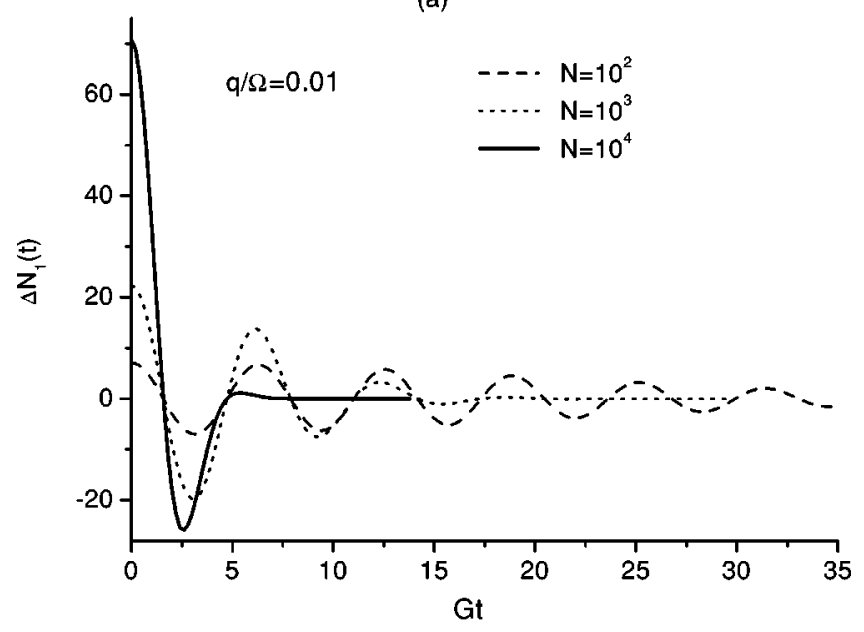

(b)

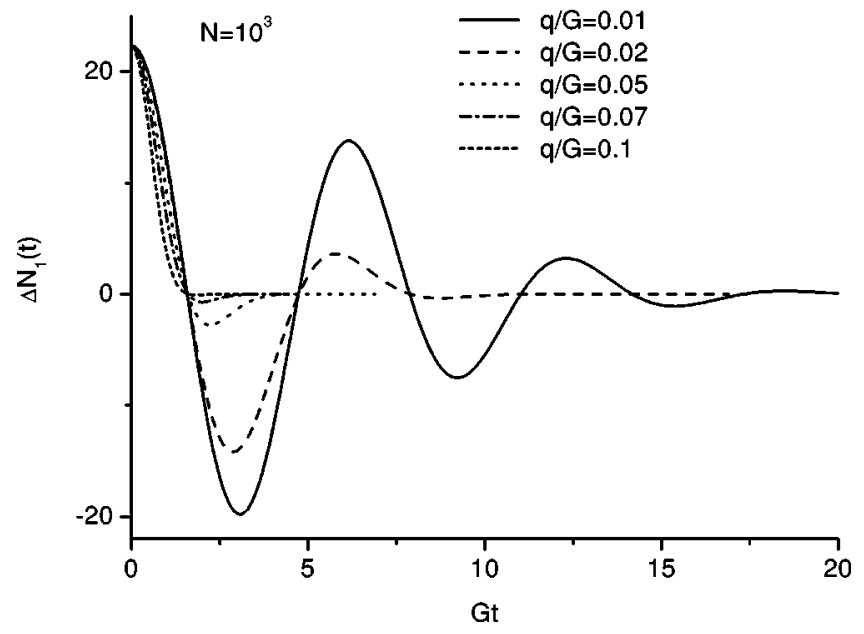

FIG. 1. Time evolution of population imbalance from initial state $\left|\psi_{1}(0)\right\rangle=|j, j\rangle, \varphi(t)=0$. (a) $q / G=0.01 ; N=10^{2}, 10^{3}, 10^{4}$. (b) $N=10^{3}, q / G=0.01 \sim 0.1$.

with and without the nonlinear interatom collisions [see Eq. (52)]. The phase-locking state is achieved again by seeing that

$$
\left\langle\hat{J}_{+}(q, t)\right\rangle_{2}\left(\phi=0, \theta=\frac{\pi}{2}\right)=\frac{N}{2} e^{-i \varphi(t)},
$$

which is the same as Eq. (46) for the case of $q=0$. In other words, phase locking is independent of the nonlinear interaction. The expectation value $\left\langle\hat{J}_{+}(q, t)\right\rangle_{2}$ for general $\phi, \theta$ is also derived analytically with the help of Eqs. (16) and (19). The resulting formula is tedious and may not be of interest to be presented here.

\section{CONCLUSION}

Using the exact solution of the time-dependent Schrödinger equation the population imbalance and phase dynamics are evaluated with various initial states, particularly with SU(2) coherent states which are the most realistic initial states for the two-species BEC created by coupling two hy- 

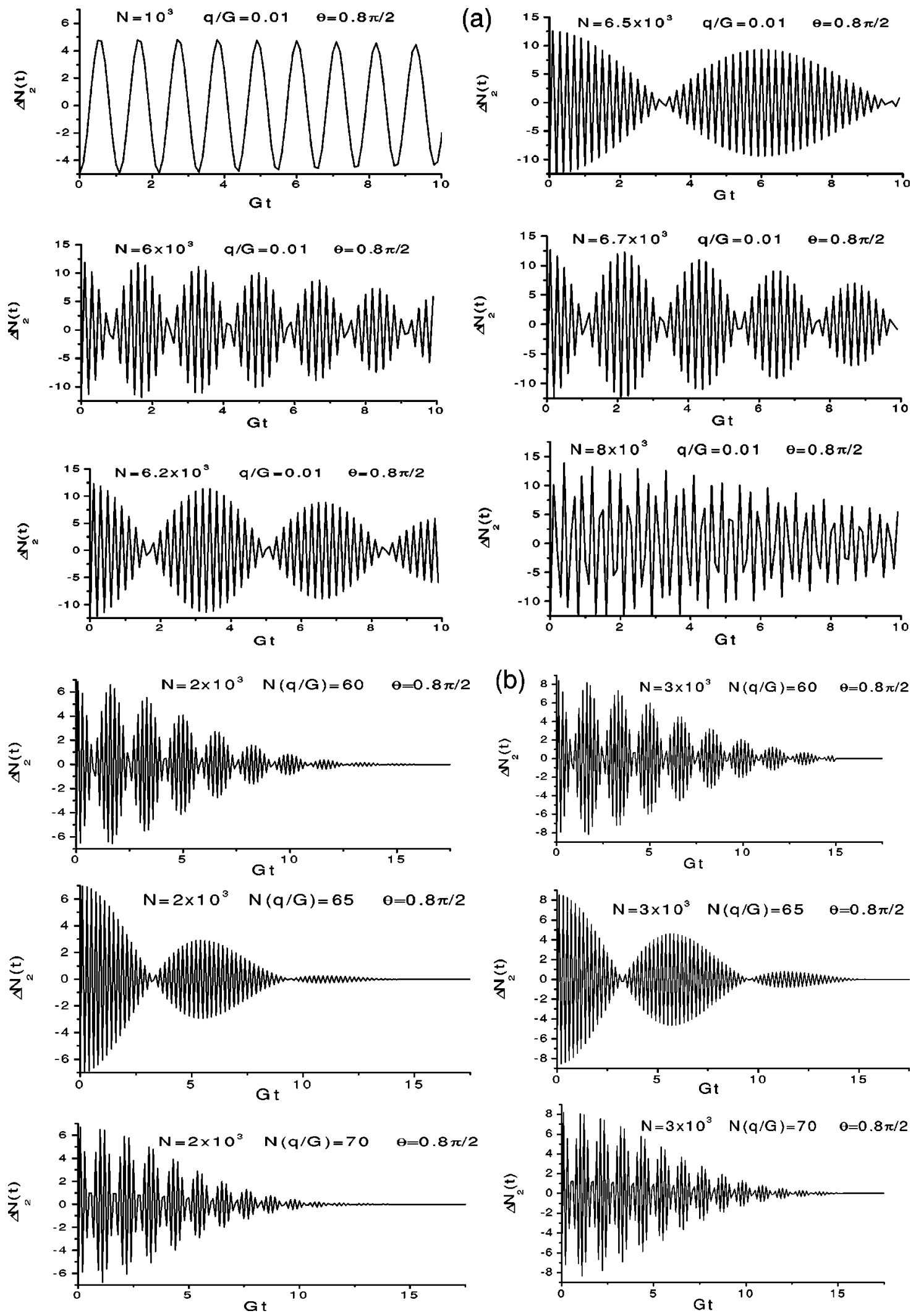

FIG. 2. Time evolution of population imbalance from initial state $\left|\psi_{2}(0)\right\rangle=|\mathbf{n}\rangle, \varphi(t)=\phi=0, \theta=0.8 \pi / 2$. (a) $q / G=0.01, N=10^{3}$ $\sim 8 \times 10^{3}$. (b) $N q / G$ dependence of $\Delta N_{2}(q, t)$, for $N q / G=60 \sim 70$; left $N=2 \times 10^{3}$; right $N=3 \times 10^{3}$. (c) $N q / G$ dependence of $\Delta N_{2}(q, t)$, for $N q / G=60 \sim 70$; left $N=4 \times 10^{3}$; right $N=5 \times 10^{3}$. 

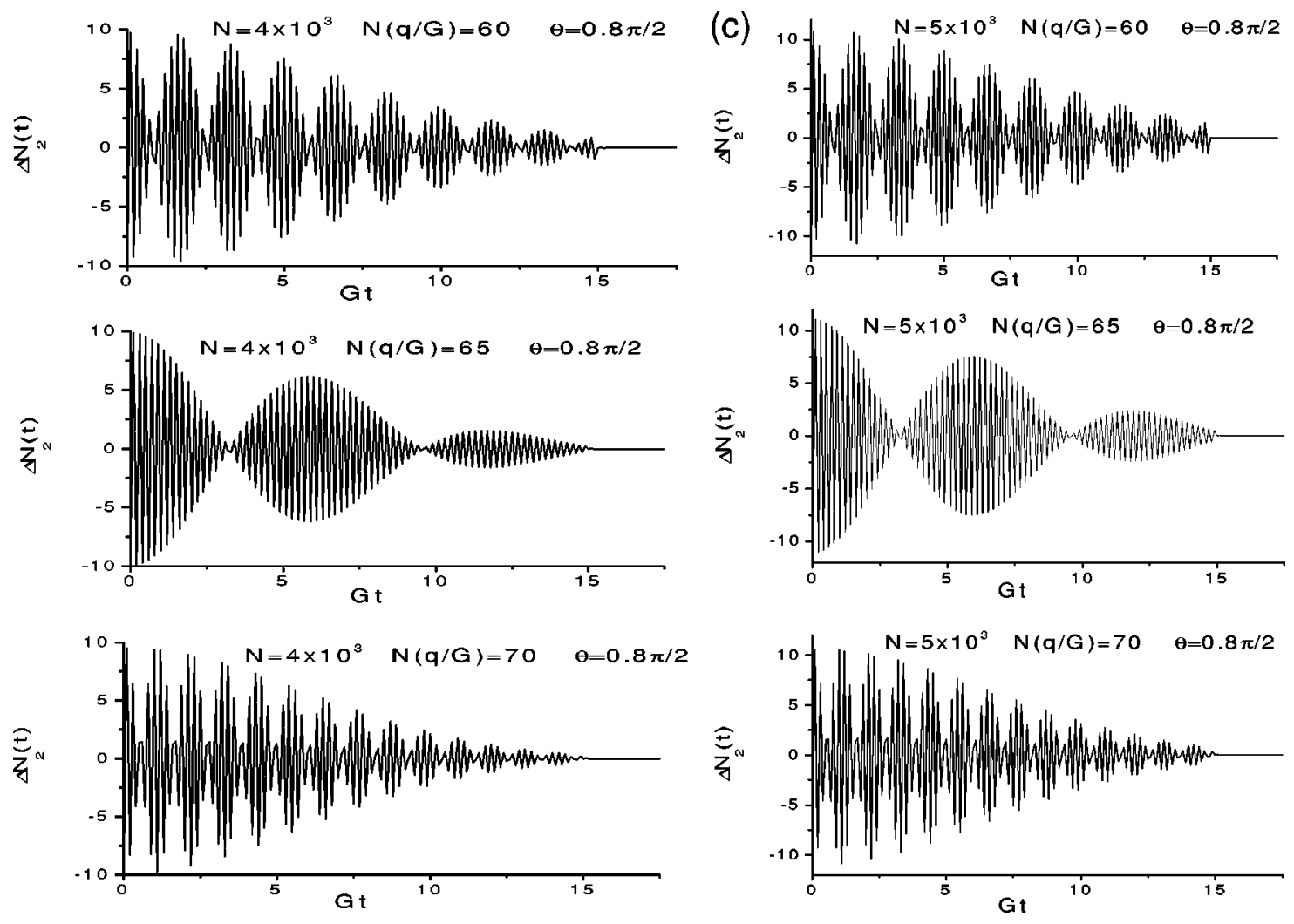

FIG. 2 (Continued).

perfine states of atoms with radiation field [12]. We conclude that self-trapping can be achieved from the initial SU(2) coherent state with an asymmetric trap potential only. Phase locking is obtained also from the initial SU(2) coherent state and is independent of the nonlinear interaction, which may be observed experimentally in terms of Ramsey's method measuring the relative phase of two components of BEC in different hyperfine atomic states. The nonlinear interatom coupling does not affect the Berry phase but leads to the damping and collapse revival of the population-imbalance oscillations. The interesting particle number dependence of the collapse revival and Berry phase as well is explored.

\section{ACKNOWLEDGMENT}

This work was supported by the Natural Science Foundation of China under Grant No. 10075032.

\section{APPENDIX}

It is easy to prove the following useful relations [20]:

$$
\begin{gathered}
\hat{R} \hat{J}_{+} \hat{R}^{\dagger}=\hat{J}_{+} \cos ^{2} \frac{\lambda}{2}-\hat{J}_{-} e^{-2 i \varphi(t)} \sin ^{2} \frac{\lambda}{2}-\hat{J}_{z} e^{-i \varphi(t)} \sin \lambda, \\
\hat{R} \hat{J}_{-} \hat{R}^{\dagger}=\hat{J}_{-} \cos ^{2} \frac{\lambda}{2}-\hat{J}_{+} e^{2 i \varphi(t)} \sin ^{2} \frac{\lambda}{2}-\hat{J}_{z} e^{i \varphi(t)} \sin \lambda,
\end{gathered}
$$

$$
\begin{aligned}
\hat{R} \hat{J}_{z} \hat{R}^{\dagger}=\hat{J}_{z} \cos \lambda+\frac{1}{2}\left(\hat{J}_{+} e^{i \varphi(t)}+\hat{J}_{-} e^{-i \varphi(t)}\right) \sin \lambda \\
i \hat{R} \frac{\partial}{\partial t} \hat{R}^{\dagger}=2 \frac{d \varphi}{d t} \sin ^{2} \frac{\lambda}{2} \hat{J}_{z}-\frac{1}{2} \frac{d \varphi}{d t} \sin \lambda\left(e^{i \varphi} \hat{J}_{+}+e^{-i \varphi} \hat{J}_{-}\right) \\
+\frac{i}{2} \frac{d \lambda}{d t}\left(e^{i \varphi} \hat{J}_{+}-e^{-i \varphi \hat{J}_{-}}\right) .
\end{aligned}
$$

The time-dependent angular momentum operators $\hat{J}_{z}$ and $\hat{J}_{+}$are obtained, respectively, as

$$
\begin{aligned}
\hat{J}_{z}(t)= & \hat{U}^{\dagger}(t) \hat{J}_{z} \hat{U}(t) \\
= & {\left[\cos ^{2} \lambda+\sin ^{2} \lambda \cos \{\alpha t+\varphi(t)\}\right] \hat{J}_{z} } \\
& +\frac{1}{2} \sin \lambda\left[-\cos \lambda+\cos ^{2} \frac{\lambda}{2} e^{-i[\alpha t+\varphi(t)]}\right. \\
& \left.-\sin ^{2} \frac{\lambda}{2} e^{i[\alpha t+\varphi(t)]}\right] \hat{J}_{+}+\frac{1}{2} \sin \lambda \\
& \times\left[-\cos \lambda+\cos ^{2} \frac{\lambda}{2} e^{i(\alpha t+\varphi(t))}\right. \\
& \left.-\sin ^{2} \frac{\lambda}{2} e^{-i(\alpha t+\varphi(t))}\right] \hat{J}_{-}
\end{aligned}
$$




$$
\begin{aligned}
\hat{J}_{+}(t)= & \sin \lambda\left[\cos ^{2} \frac{\lambda}{2} e^{i \alpha(t)}-\sin ^{2} \frac{\lambda}{2} e^{-i[\alpha(t)+2 \varphi(t)]}-\cos \lambda e^{-i \varphi(t)}\right] \hat{J}_{z}+\left[\cos ^{4} \frac{\lambda}{2} e^{i \alpha(t)}+\sin ^{4} \frac{\lambda}{2} e^{-i[\alpha(t)+2 \varphi(t)]}\right. \\
& \left.+\frac{1}{2} \sin ^{2} \lambda e^{-i \varphi(t)}\right] \hat{J}_{+}+\left\{-\cos ^{2} \frac{\lambda}{2} \sin ^{2} \frac{\lambda}{2}\left[e^{i \alpha(t)}+e^{-i[\alpha(t)+2 \varphi(t)]}\right]+\frac{1}{2} \sin ^{2} \lambda e^{-i \varphi(t)}\right\} \hat{J}_{-}
\end{aligned}
$$

[1] M.R. Andrews, C.G. Townsend, H.-J. Miesner, D.S. Durfee, D.M. Kurn, and W. Ketterle, Science 275, 637 (1997).

[2] D.S. Hall, M.R. Matthews, J.R. Ensher, C.E. Wieman, and E.A. Cornell, Phys. Rev. Lett. 81, 1543 (1998).

[3] D.S. Hall, in Bose-Einstein Condensates and Atom Lasers, edited by S. Martellucci, A.N. Chester, A. Aspect, and M. Inguscio, (Kluwer, New York, 2000), p. 31.

[4] G.J. Milburn, J. Corney, E.M. Wright, and D.F. Walls, Phys. Rev. A 55, 4318 (1997).

[5] A. Smerzi, S. Fantoni, S. Giovanazzi, and S.R. Shenoy, Phys. Rev. Lett. 79, 4950 (1997).

[6] S. Raghavan, A. Smerzi, S. Fantoni, and S.R. Shenoy, Phys. Rev. A 59, 620 (1999).

[7] J. Williams, R. Walser, J. Cooper, E. Cornell, and M. Holland, Phys. Rev. A 59, R31 (1999).

[8] S. Raghavan, A. Smerzi, and V.M. Kenkre, Phys. Rev. A 60, R1787 (1999).

[9] J. Javanainen and M.Y. Ivanov, Phys. Rev. A 60, 2351 (1999).

[10] M.J. Steel and M.J. Collett, Phys. Rev. A 57, 2920 (1998).

[11] A. Smerzi and S. Raghavan, Phys. Rev. A 61, 063601 (2000).

[12] D. Gordon and C.M. Savage, Phys. Rev. A 59, 4623 (1999).

[13] L.-M. Kuang and Z.-W. Ouyang, Phys. Rev. A 61, 023604
(2000).

[14] W.D. Li, X.J. Zhou, Y.Q. Wang, J.Q. Liang, and W.M. Liu, Phys. Rev. A 64, 015602 (2001).

[15] N. Ramsey, Molecular Beams (Claredon Press, Oxford, 1956).

[16] I. Fuentes-Guridi, J. Pachos, S. Bose, V. Vedral, and S. Choi, Phys. Rev. A 66, 022102 (2002).

[17] M.V. Berry, Proc. R. Soc. London, Ser. A 392, 45 (1984).

[18] A. Perelomov, Generalized Coherent States and Their Applications (Springer, Heidelberg, 1986).

[19] J.-Q. Liang and H.J.W. Müller-Kirsten, Ann. Phys. (N.Y.) 219, $42(1992)$

[20] Y.-Z. Lai, J.-Q. Liang, H.J.W. Müller-Kirsten, and J.-G. Zhou, Phys. Rev. A 53, 3691 (1996).

[21] A. Inomata, H. Kuratsuji, and C.C. Gerry, Path Integrals and Coherent States of $S U(2)$ and $S U(1,1)$ (World Scientific, Singapore, 1992).

[22] D. Loss, D.P. DiVincenzo, and G. Grinstein, Phys. Rev. Lett. 69, 3232 (1992).

[23] J.-Q. Liang, H.J.W. Müller-Kirsten, and J.-G. Zhou, Z. Phys. B: Condens. Matter 102, 525 (1997).

[24] Z.-D. Chen, J.-Q. Liang, and F.-C. Pu, Phys. Lett. A 300, 654 (2002). 